\title{
Characterizing Ireland's wave energy resource
}

\section{Brendan Cahill}

Hydraulics \& Maritime Research Centre, UCC

\section{Introduction}

In theory, the energy that could be extracted from the oceans is well in excess of any current, or future, human requirements. While wave energy currently lags behind conventional, carbon based sources of power and other renewable sources of energy such as wind and solar, advances continue to be made. The developers of Wave Energy Converters (WECs), the devices that are designed to harness the power of ocean waves, require methods to compare and evaluate the wave energy resource at different locations in order to allow them to select the most suitable sites to achieve optimal power capture and economic performance from their installations. The focus of my Ph.D. research is towards developing new methods for characterizing the wave energy resource off the west coast of Ireland with reference to the potential power available and the performance of typical devices, and also to allow for the comparison of possible sites for the development of WEC projects. In particular, efforts are being made to differentiate between which sets of wave conditions, known as sea states, occur most frequently at a location of interest and which sea states contribute most to the energy that can be expected to be produced by a WEC.

\section{Wave measurement and data processing}

In order to quantify and characterize the ocean energy resource at a location of interest, it is first necessary to collect data which describes the nature of the incident waves. Many different wave measurement techniques are in use worldwide, each with their own inherent advantages and weakness. In the past, wave heights were estimated visually by a network of trained observers aboard ships, a method which produces surprisingly accurate results, notwithstanding the human capacity for exaggeration! Nowadays a plethora of tools are available to ocean researchers, ranging from pressure sensors fixed to the sea bed to satellite mounted radar systems.

This research relies on data collected by wave buoys (Fig. 1); one of the most commonly used measurement systems in the field of ocean energy. These buoys are moored to the seabed and employ complex instrumentation to measure the motion of the passing waves. 


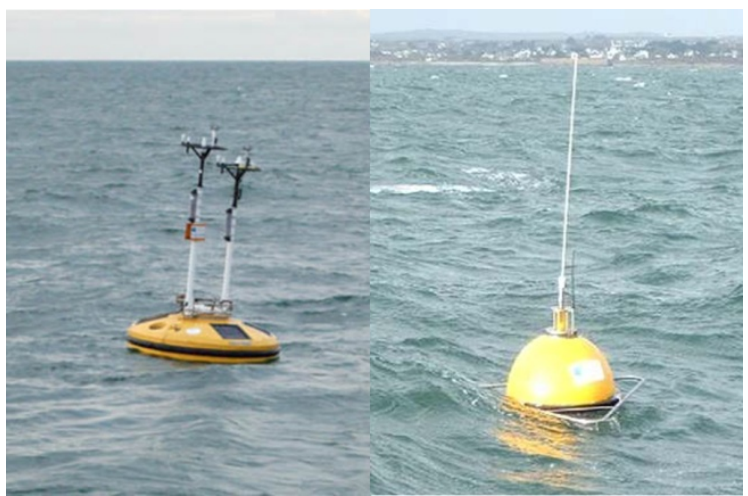

Figure 1: Wave measurement buoys: (a) Fugro Wavescan buoy, (b) Datawell Waverider buoy

This data is then transmitted to an onshore receiving station. A typical 30 minute recording of wave elevation, measured at the Galway Bay Wave Energy test site, is shown in Fig. $2 \mathrm{~b}$.

An important aspect of my project to date has been the implementation of an efficient computer program to collate the collected wave buoy measurements, apply quality control checks to remove erroneous recordings and finally analyse the data in order extract useful information. This final step can be difficult. Visual investigation of the wave record illustrated in Fig. 2b is almost impossible; it appears to be a very confused and irregular signal, made up of many waves with different heights. Advanced analysis techniques must be applied to simplify the data by decomposing this surface elevation profile into its corresponding wave spectrum, illustrated in Fig. 2a.The size and shape of this spectrum

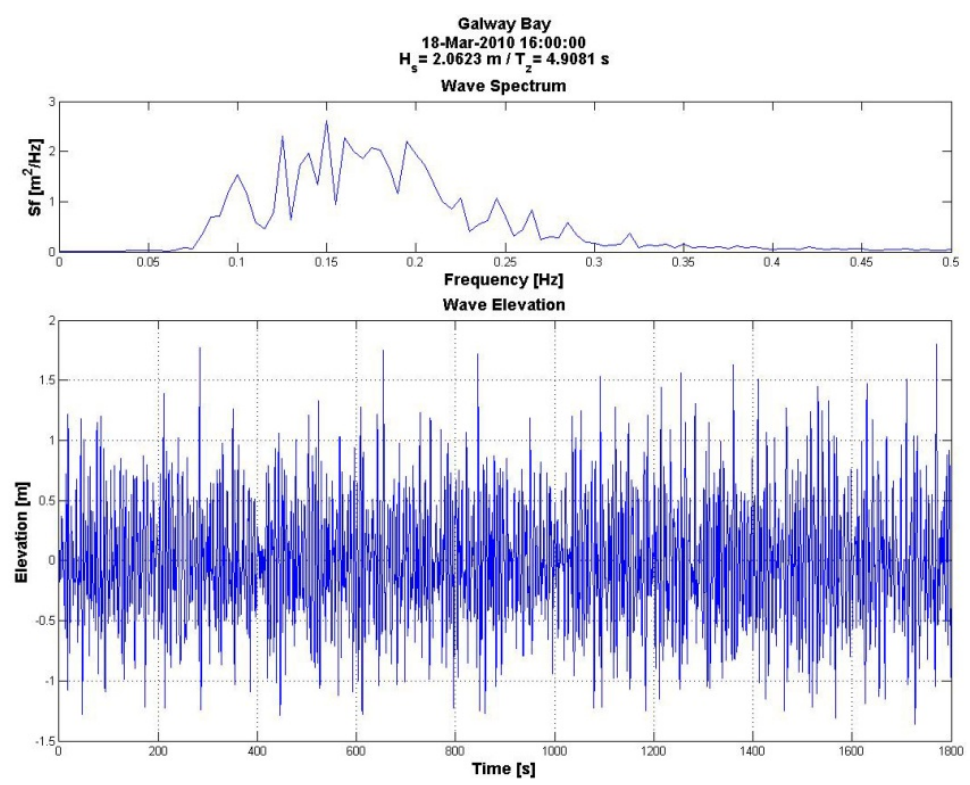

Figure 2: (a) Wave spectrum corresponding to (b) 30min recording of wave elevation from Galway Bay 
provides us with details of the character of the observed wave conditions and allows for the calculation of important parameters used in the description of wave climate. These parameters include the significant wave height $(\mathrm{Hm} 0)$, average height of the largest third of all the observed waves in a record, and the wave period (T02), the time it takes for two successive wave crests to pass a fixed point in space.

\section{Resource characterization}

\section{The need for resource characterization}

The accurate characterization of the wave energy resource will be vital for all stages of the lifecycle of a commercial wave farm. At the initial design phase it will be necessary in order to quantify the amount of power that an individual device can be expected to produce and predict the wave induced forces it will need to withstand. Resource characterization will also be required to ensure that WEC developments are sited at the most energy rich and economically viable locations. Finally, once these devices are deployed at sea and connected to the electrical grid, knowledge of the incident wave conditions will allow for the monitoring of the performance of the farm. My research aims at aiding this process by identifying suitable methods for the analysis of measured wave data in order to describe and characterize the wave climate that can be expected at a site.

\section{Analysis and results}

One of the most useful and commonly employed techniques for visualising the wave energy resource at a location of interest is to create graphics known as scatter plots, which illustrate the occurrence of particular combinations of wave parameters such as $\mathrm{Hm} 0$ and T02, referred to as sea states. These scatter plots provide a useful method of ascertaining an overall understanding of the wave resource. Fig. 3illustrates range the sea states experienced through the year 2010 at the Atlantic Marine Energy Test Site (AMETS), a grid connected site for the deployment of pre-commercial WECS that is being developed by the Sustainable Energy Authority of Ireland (SEAI) near Belmullet, Co. Mayo, based on wave data processed using the methods outlined in the previous section. The percentage occurrence of different combination of $\mathrm{Hm} 0$ and $\mathrm{T} 02$ is noted in the relevant box and along with the colour shading indicates how frequently each sea state occurs. Fig. 3 shows that WECs deployed at AMETS could expect to experience a wide spread of conditions, with significant wave heights greater then $10 \mathrm{~m}$ evident during storms. The presence of a red/orange hotspot is also noticeable, highlighting that for most of the year wave conditions fell within a significant wave height range of 1-3 metres and a wave period range of 5-8 seconds. In the past, these sea states have been deemed to be the most significant for the capture of wave energy. 


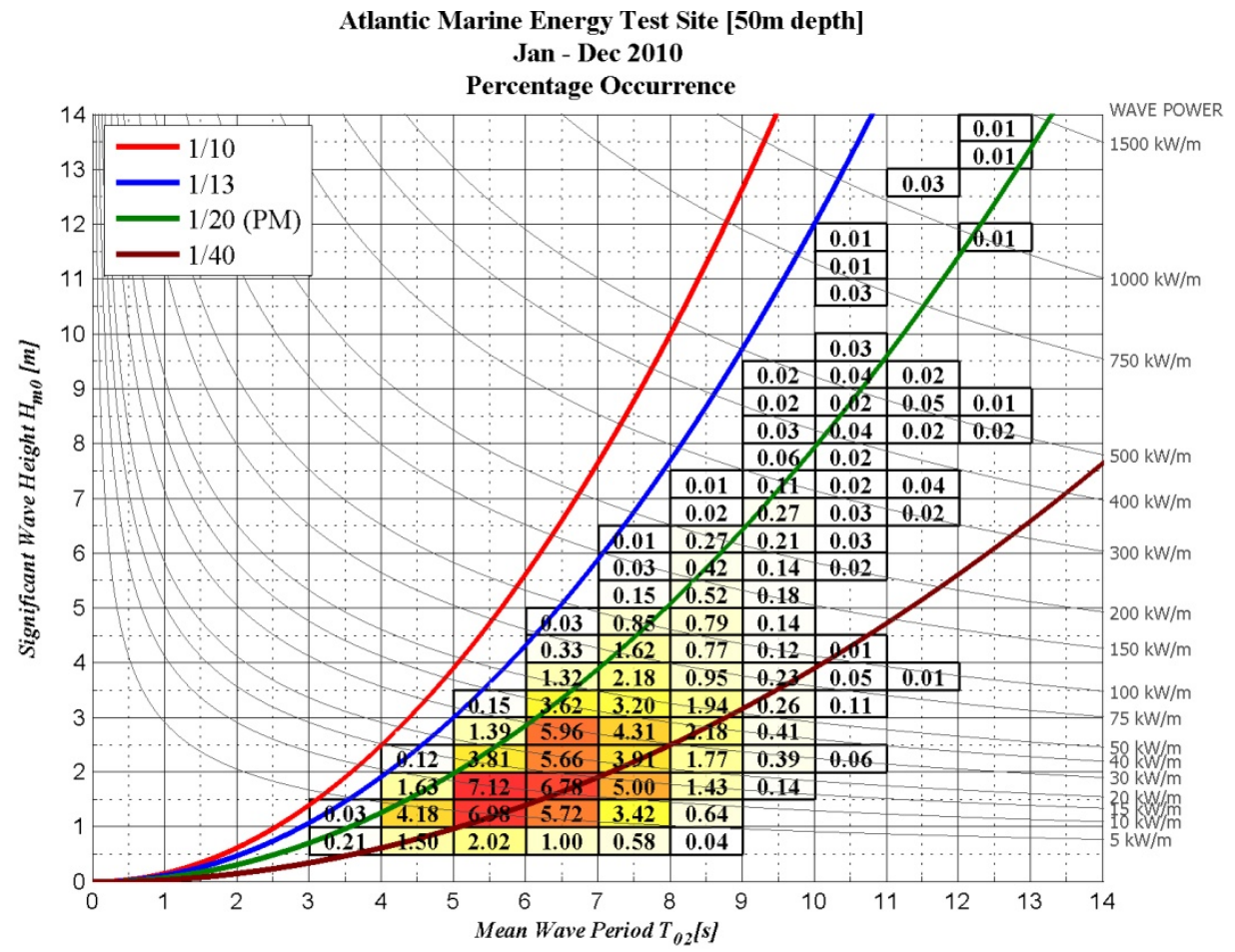

Figure 3: Sea State occurrence (\%) scatter plot for Atlantic Marine Energy Test Site for 2010

An important finding from my research has been to identify a major limitation associated with scatter plots like Fig. 3 in that they fail to fully describe which sea states contribute most to the wave energy experienced at a particular location. The AMETS scatter diagram is redrawn in Fig. 4, using analysis techniques developed for this project, with percentage occurrence replaced by the percentage contribution that each sea state makes to total incident wave energy over the course of the year. When this diagram is compared with Fig. 3 it is evident that there is an obvious upward shift in the position of the hotspot. This indicates that the most frequently occurring conditions over the course of a year are not necessarily the most energetic, and that the sea states highlighted in Fig.4 are in fact more favourable for the capture of power.

Generally WECS are not able to operate efficiently in all the wave conditions they will experience and must be tuned to achieve optimal performance across a limited range of sea states. As my research has shown, two significant clusters of sea states exist at a site: the most frequently occurring conditions and the most energy rich, with the latter group proving to be the most significant if maximum power capture is desirable. These findings should assist the designers of WECs in deciding on the sea states in which their device should achieve optimum performance in and in selecting the most favourable locations for deploying their wave farms. 


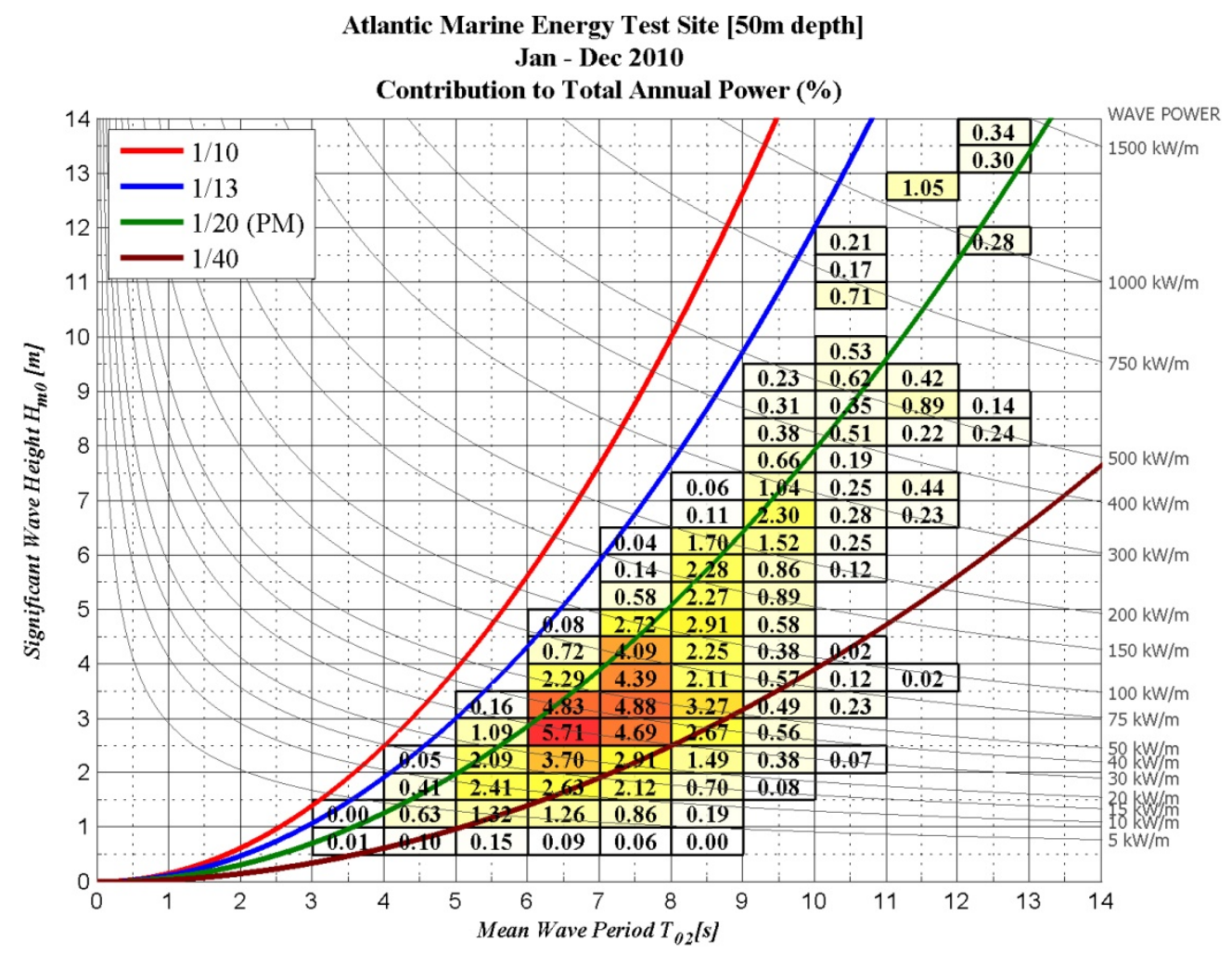

Figure 4: Sea State contribution to total annual energy (\%) scatter plot for Atlantic Marine Energy Test Site for 2010

\section{Conclusion}

This paper has described the wave data processing and analysis techniques that are being developed as part of this Ph.D. project in order to characterise the wave energy resource off the Irish coast. It has been demonstrated that the most prevalent wave conditions experienced at a typical exposed ocean location are not the most energy rich when observed over the course of a year. Future research will focus on quantifying the level of variability that exists in the wave energy resource at various sites and will build on these results to propose a methodology which will allow WEC developers to select the most appropriate deployment locations and wave conditions for their particular devices.

The author wishes to thank Dr. Tony Lewis for his supervision and assistance and the Irish Research Council for Science, Engineering and Technology (IRCSET) for funding this research. 